\title{
Fast georeferencing/geocoding tool for old maps with large distortions
}

\author{
Gede, $\mathrm{M}^{\mathrm{a}, *}$ \\ ${ }^{a}$ ELTE Eötvös Loránd University, Budapest, Hungary, saman@map.elte.hu \\ * Corresponding author
}

Keywords: georeferencing, distorted old maps, parallel view

\begin{abstract}
:
Maps created before the $17^{\text {th }}$ century often have large distortions which make it useless to force them into any modern map projection by georeferencing. In a local scope, however, they preserve spatial relationships between map objects, therefore, using an appropriate control point set and local interpolation it is possible to define a quite accurate connection between the old and a modern map.

Additionally, researchers in the past decades created long lists of settlements of these maps, often without any geometric information but matching most places with present day settlements.

The author developed a web application to help geocoding these lists and at the same time to create an accurate georeferenced of the corresponding old maps. This tool displays the old map and a recent web map parallel, without forcing the projection of the web map to the old one. The user can load settlement lists, perform a bulk geocoding based on present day names. The geocoded places appear on the new map, and any of these points can be also placed on the old map, defining a control point pair. After setting enough control points, all the other place names can be automatically placed by local interpolation based on the control points. The place positions can be refined manually by the user, which will improve the accuracy of the automatic placement as well.
\end{abstract}

